\title{
encyclopedia on ad hoc and ubiquitous computing
}

theory and design of wireless ad hoc, sensor, and mesh networks 
This page intentionally left blank 


\title{
encuclopedia on ad hoc and ubiquitous computing
} theory and design of wireless ad hoc, sensor, and mesh networks

\author{
edited by \\ dharma $p$ agrawal $\&$ bin xie \\ university of cincinnati, usa
}




\section{Published by}

World Scientific Publishing Co. Pte. Ltd.

5 Toh Tuck Link, Singapore 596224

USA office: 27 Warren Street, Suite 401-402, Hackensack, NJ 07601

UK office: 57 Shelton Street, Covent Garden, London WC2H 9HE

\section{British Library Cataloguing-in-Publication Data}

A catalogue record for this book is available from the British Library.

\section{ENCYCLOPEDIA ON AD HOC AND UBIQUITOUS COMPUTING \\ Theory and Design of Wireless Ad Hoc, Sensor, and Mesh Networks}

Copyright @ 2010 by World Scientific Publishing Co. Pte. Ltd.

All rights reserved. This book, or parts thereof, may not be reproduced in any form or by any means, electronic or mechanical, including photocopying, recording or any information storage and retrieval system now known or to be invented, without written permission from the Publisher.

For photocopying of material in this volume, please pay a copying fee through the Copyright Clearance Center, Inc., 222 Rosewood Drive, Danvers, MA 01923, USA. In this case permission to photocopy is not required from the publisher.

Desk Editor: Tjan Kwang Wei

ISBN-13 978-981-283-348-8

ISBN-10 981-283-348-X

Typeset by Stallion Press

Email: enquiries@stallionpress.com

Printed in Singapore. 


\title{
Preface
}

\begin{abstract}
About the Book
With an explosive growth of wireless communication devices, ad hoc and ubiquitous computing technologies have received unparallel attention from both the academia and industry. In recent years, a number of significant advances have been made in these technologies, with the target of offering more exciting and efficient services for different application scenarios, at anytime and anywhere. These advances have ranged from the development of Mobile Ad Hoc Networks (MANETs) for disaster applications, embedded wireless sensor networks for monitoring applications, and startling discovery of wireless mesh networks that promises to ensure high bandwidth Internet access to mobile users by using multi-hop, multi-channel, and multi-radio technology. These recent developments not only have kindled considerable interest in the study of different ubiquitous computing technologies, but also have fostered unusual civilian, industrial, and military applications. The intent of this book is to elaborate on the fundamental concepts, protocols, and algorithms, to provide a comprehensive understanding of this area of ubiquitous computing and to extend its usefulness to much higher heights.
\end{abstract}

\section{Coverage of Topics}

Due to diversified application scenarios, that necessitate different performance requirements in deploying these technologies, design issues for ubiquitous computing are extremely complicated and there are a number of technical challenges that need to be explored, involving every layer of the OSI (Open Systems Interconnection) protocol stack. This book has a total of 23 chapters, dealing with MANETs, wireless sensor networks, and wireless mesh networks. Design and implementation of MANET are also essential for the other two network paradigms and thus, we begin by illustrating first 8 chapters of MANETs dealing with different subject areas. Then, the design, application, and security issues in wireless sensor networks are discussed from Chapter 9 to Chapter 17. Finally, many underlying characteristics 
and associated issues of wireless mesh networks are covered from Chapter 18 to Chapter 23.

\section{A. Advances in MANET}

MANETs have been successfully explored for many applications such as battlefield communication and emergency response due to underlying capability of fast deployment in a scenario that resorts to quick communication, and has become a popular research area since mid 1990s. Substantial research efforts have been made in this area, and we have selected eight active topics as our prime interest, including (i) Link Quality Models, (ii) Scalable Multicast Routing Protocols, (iii) TCP, Congestion, and Admission Control Protocols for Multi-hop Transmission, (iv) Directional Antennas for MANET, (v) Peer to Peer and Content Sharing in Vehicular Ad Hoc Networks, (vi) Properties of the Vehicle to Vehicle Channel for Dedicated Short Range Communications, (vii) Radio Resource Management in Cellular Relay Networks, and (viii) Game Theoretic Tools applied to Wireless Networks. These subjects depict fundamental concepts in communication, or reflect the recent research trends in MANETs. For example, most of the designed protocols in MANETs assumed an ideal link model, with equal radio coverage in all the directions. This means that all transmitted packets can be successfully received if the receiver is located with the disk area of radius R. However, in 2003, the Berkeley mote platform has demonstrated that the radio signals to be irregular in nature, which results in variable packet losses in different directions. This has motivated many researchers to consider new link models that could accurately represent the link quality, and accordingly introduce reliable routing protocols. In the past decade, Vehicular Ad Hoc Networks (VANET), that specialize MANET to vehicle to vehicle $(\mathrm{V} 2 \mathrm{~V})$ and vehicle to infrastructure (V2I) communications have received a great deal of attention by the research community to offer ubiquitous and pervasive network as a part of our daily life. To provide a complete understanding, we have scheduled two chapters to address the recent research activities in this emerging area.

\section{B. Issues in wireless sensor networks}

Design of wireless sensor networks was initially motivated by military applications such as battlefield surveillance. However, in the past few years, it has been the focus of many studies for numerous civilian and industrial applications, such as environment and habitat monitoring, object tracking, healthcare, home automation, and traffic control. In this book, we have included nine chapters in the area to address (i) Sensor Routing Protocols, (ii) Quality of Service (QoS) for Sensor Traffic, (iii) Mobility in wireless sensor networks, (iv) Delay-Tolerant Mobile Sensor Networks, (v) Integration of Radio frequency identification (RFID) and wireless sensor networks, (vi) Integrating Sensor Networks with the Semantic Web, (vii) Effective Multi-user Broadcast Authentication in Wireless Sensor, (viii) Wireless Sensor 
Network Security, and (ix) Information Security. Unlike MANETs, routing protocols for wireless sensor networks has to be optimized such that it can not only efficiently route the packets with minimum overheads, but also consider severe power constraints. One chapter is designed to highlight architectural and operational protocols for QoS-enabled traffic in sensor networks. RFID facilitates detection and identification of objects that are not easily detectable or distinguishable by using current sensor technologies. A chapter is designed to present RFID technology and illustrates recent research works, new patents, academic products and applications that integrate RFID with sensor networks. On the other hand, semantic web represents a spectrum of effective technologies that support complex, cross-jurisdictional, heterogeneous, dynamic and large scale information systems and growing research efforts on integrating sensor networks with semantic web technologies have led to a new frontier in networking and data management research. With respect to security, three chapters are designed to illustrate security attacks, authentication, and information security in wireless sensor networks.

\section{Characteristics of wireless mesh networks}

Wireless mesh networks employ multi-channel and multi-radio in a static MANET with the integration of the Internet infrastructure to support cost-efficient and high bandwidth Internet accessibility for mobile users. The network coverage can span a community, an enterprise, or an entire city to support a broad range of applications such as voice, data transfer, video delivery, home networking, etc. Within a short span of time, wireless mesh network technology has stirred considerable interest in both the commercial and academic spheres, and consequently, the consideration of wireless mesh network technology is indispensable in this book. The topics addressed in the book includes: (i) Wireless Mesh Network Architecture, (ii) Multihop Medium Access Control (MAC) design and implementation, (iii) Multi-channel Channel Assignment, (iv) Multi-hop, Multi-path, and Load Balancing Routing, (v) Mobility Management, and (vi) Selfishness and Security. In contrast to peer to peer structure of a MANET, a wireless mesh network constitutes a wireless backhaul with static mesh routers which serves as the wireless access points for mobile users. Therefore, deployment of wireless mesh networks is substantially different from MANETs, and details are given on the chapters of wireless mesh network architecture. It may be noted that in spite of sharing some common characteristics with MANETs, wireless mesh networks have their unique design issues such as multi-channel MAC, multi-path routing, traffic patterns, and fairness, etc. We address these topics to cover significant characteristics of wireless mesh networks.

\section{The Use of the Book}

This book provides a comprehensive reference material for students, instructors, researchers, engineers, and other professionals in building their understanding of 
ad hoc and ubiquitous computing. The book is collective efforts of many professional experts and researchers. Each chapter on a particular topic is written in a tutorial manner and the chapters are organized so that the topics covered are easy to understand. On the other hand, each chapter is self-contained and illustrations are independent of other chapters. Therefore, the topics can be selected as per individual interest. Each chapter has about 10 questions that cover significant points of the chapter. You can find answers to selected questions at our website. In addition, slides are available at our website that can be useful for teaching and presentation by the instructors. Our publisher encouraged us to prepare this volume and we hope the efforts could prove to be useful for the readers.

Dharma Prakash Agrawal Bin Xie Cincinnati 


\section{Contents}

Preface $\quad$ v

Part 1: Mobile Ad hoc Networks 1

1. Survey on Link Quality Models in Wireless Ad Hoc Networks 3

M. Lu and J. Wu

2. Scalable Multicast Routing in Mobile Ad Hoc Networks

R. Menchaca-Mendez and J. J. Garcia-Luna-Aceves

3. TCP, Congestion and Admission Control Protocols in Ad Hoc Networks

A. Mishra, B. Awerbuch and R. Cole

4. Wireless Ad Hoc Networks with Directional Antennas

B. Alawieh, C. Assi and H. Mouftah

5. Peer-to-Peer and Content Sharing in Vehicular Ad Hoc Networks M. Abuelela and S. Olariu

6. Properties of the Vehicle-to-Vehicle Channel for Dedicated Short Range Communications

L. Cheng, B. E. Henty, D. D. Stancil and F. Bai 
7. Radio Resource Management in Cellular Relay Networks K.-D. Lee and V. C. M. Leung

8. Game Theoretic Tools Applied to Wireless Networks H. Liu, B. Krishnamachari and S. Kapadia

\section{Part 2: Wireless Sensor Networks}

9. Wireless Sensor Networks - Routing Protocols

A. Jamalipour and M. A. Azim

10. Handling QoS Traffic in Wireless Sensor Networks

M. Younis, K. Akkaya and M. Youssef

11. Mobility in Wireless Sensor Networks

A. Asok, K. M. Sivalingam and P. Agrawal

12. Delay-Tolerant Mobile Sensor Networks

Y. Wang and $H$. Wu

13. Integration of RFID and Wireless Sensor Networks

H. Liu, M. Bolic, A. Nayak and I. Stojmenovi

14. Integrating Sensor Networks with the Semantic Web

Y. Pei and B. Wang

15. Effective Multi-User Broadcast Authentication in Wireless

Sensor Networks

K. Ren, W. Lou and Y. Zhang

16. Security Attacks and Challenges in Wireless Sensor Networks A.-S. K. Pathan and C. S. Hong

17. Information Security in Wireless Sensor Networks A. Ouadjaout, M. Bagaa, A. Bachir, Y. Challal, N. Lasla and L. Khelladi 
Part 3: Wireless Mesh Networks

18. Network Architecture and Flow Control in Multi-Hop Wireless

Mesh Networks

D. Nandiraju, N. Nandiraju and D. P. Agrawal

19. Multi-hop MAC: IEEE 802.11s Wireless Mesh Networks

501

R. C. Carrano, D. C. M. Saade, M. E. M. Campista,

I. M. Moraes, C. V. N. de Albuquerque, L. C. S. Magalhães,

M. G. Rubinstein, L. H. M. K. Costa and O. C. M. B. Duarte

20. Channel Assignment in Wireless Mesh Networks

W. Fu, B. Xie and D. P. Agrawal

21. Multi-hop, Multi-path and Load Balanced Routing in Wireless Mesh Networks

S. Mishra and N. Shenoy

22. Mobility Management in Wireless Mesh Networks

P. Wu, B. Landfeldt and A. Y. Zomaya

23. Selfishness and Security Schemes for Wireless Mesh Network

601

L. Santhanam, B. Xie and D. P. Agrawal

Solutions

631

Biography 